\title{
Investigating Origins of Ventricular Arrhythmia Arising from Right Ventricular Outflow Tract and Comparing Initial Ablation Strategies
}

\author{
Zhi Jiang ${ }^{1}$, LIU Qifang${ }^{1}$, Ye Tian ${ }^{1}$, Yidong Zhao ${ }^{2}$, Wei Liu ${ }^{1}$, Longhai Tian ${ }^{1}$, Jing Huang ${ }^{1}$, \\ Shui Tian ${ }^{1}$, Yaxi Zheng ${ }^{1}$, Jason $\mathrm{Tri}^{3}$, and Long Yang ${ }^{1}$ \\ ${ }^{1}$ Guizhou Provincial People's Hospital \\ ${ }^{2}$ Guizhou Medical University \\ ${ }^{3}$ Mayo Clinic Minnesota
}

May 16, 2021

\begin{abstract}
Background The origin distribution of right-ventricular-outflow-tract (RVOT) ventricular arrhythmias (VAs) remains unclear. There is limited data on the ablation effectiveness of the reversed U-curve method compared with the antegrade method. Objectives To investigate the origin distribution of RVOT-type VAs and compare the ablation effectiveness of the two methods. Method Consecutive patients who had idiopathic RVOT-type VAs were prospectively enrolled. After activation mapping, patients were randomly assigned to supravalvular strategy using the reversed U-curve or subvalvular strategy using the antegrade method. The primary outcome was initial ablation (IA) success, defining as the successful ablation within the first three attempts. Results 61 patients were enrolled from November 2018 to June 2020. Activation mapping revealed 34/61 (55.7\%) of the earliest ventricular activating (EVA) sites were above the pulmonary valves (PVs). The IA success rate was $25 / 33(75.8 \%$ ) in the patients assigned to supravalvular strategy as compared with 16/28(57.1\%) in those assigned to subvalvular strategy $(\mathrm{P}=0.172)$. Logistic regression revealed a substantial and qualitative interaction between the EVA sites and IA strategies (Pinteraction $<0.001$ ). For multiple-comparison, either strategy had a remarkably higher IA success rate in treating its ipsilateral EVA sites than contralateral ones $(\mathrm{P}<0.0083)$. Conclusion Of the idiopathic RVOT-type VA origins, half were located above the PV. The two strategies did not differ in the primary outcomes. However, they complement locating the EVA sites and facilitate ipsilateral ablation, which produces a significantly higher IA success rate. (Chinese Clinical Trial Registry number, ChiCTR2000029331)
\end{abstract}

Title: Investigating Origins of Ventricular Arrhythmia Arising from Right Ventricular Outflow Tract and Comparing Initial Ablation Strategies

Short title: Comparison of Initial Ablation Strategies for RVOT-type VAs Based on Origins

Authors: Zhi Jiangabd, MBBS, PhD, Qifang Liu ${ }^{\mathrm{ab}}$, MBBS, Ye Tian ${ }^{\mathrm{ab}}$, MBBS, Yidong Zhao $^{\mathrm{c}}$, MBBS, Wei Liu $^{\text {ab }}$, MBBS, Longhai Tian ${ }^{\text {ab }}$, MBBS, Jing Huang ${ }^{\text {ab }}$, MBBS, Shui Tian ${ }^{\text {ab }}$, MBBS, Yaxi Zheng ${ }^{\text {ab }}$, MBBS, Jason Tri ${ }^{\mathrm{d}}$, Long Yang ${ }^{\mathrm{ab}}$, MBBS

Affiliations: From ${ }^{a}$ Cardiology Department, Guizhou Provincial People's Hospital, Guiyang, China; ${ }^{\mathrm{b}}$ Guizhou Provincial Cardiovascular Disease Institute, Guiyang, China; c Guizhou Medical University, Guiyang, China; ${ }^{\mathrm{d}}$ Cardiovascular Diseases, Mayo Clinic, Rochester, Minnesota, USA

Corresponding Author: Dr. Long Yang, Email: yanglong1001@outlook.com, Telephone: +86-085185922979, Fax: +86-0851-85924943 
Correspondence Address: Cardiology Department, Guizhou Provincial People's Hospital \& Guizhou Provincial Cardiovascular Disease Institute, No.83 East Zhongshan Road, Yunyan District, Guiyang, 550002, China.

Disclosure: No potential conflict of interest relevant to this article to report.

Word count: 2634

\section{Abstract}

Background The origin distribution of right-ventricular-outflow-tract (RVOT) ventricular arrhythmias (VAs) remains unclear. There is limited data on the ablation effectiveness of the reversed U-curve method compared with the antegrade method.

Objectives To investigate the origin distribution of RVOT-type VAs and compare the ablation effectiveness of the two methods.

Method Consecutive patients who had idiopathic RVOT-type VAs were prospectively enrolled. After activation mapping, patients were randomly assigned to supravalvular strategy using the reversed U-curve or subvalvular strategy using the antegrade method. The primary outcome was initial ablation (IA) success, defining as the successful ablation within the first three attempts.

Results 61 patients were enrolled from November 2018 to June 2020. Activation mapping revealed 34/61 $(55.7 \%)$ of the earliest ventricular activating (EVA) sites were above the pulmonary valves (PVs). The IA success rate was $25 / 33(75.8 \%)$ in the patients assigned to supravalvular strategy as compared with $16 / 28(57.1 \%)$ in those assigned to subvalvular strategy $(\mathrm{P}=0.172)$. Logistic regression revealed a substantial and qualitative interaction between the EVA sites and IA strategies $\left(\mathrm{P}_{\text {interaction }}<0.001\right)$. For multiplecomparison, either strategy had a remarkably higher IA success rate in treating its ipsilateral EVA sites than contralateral ones $(\mathrm{P}<0.0083)$.

Conclusion Of the idiopathic RVOT-type VA origins, half were located above the PV. The two strategies did not differ in the primary outcomes. However, they complement locating the EVA sites and facilitate ipsilateral ablation, which produces a significantly higher IA success rate. (Chinese Clinical Trial Registry number, ChiCTR2000029331)

Keywords: pulmonary valve, pulmonary sinus cusp, premature ventricular contraction, radiofrequency, activation mapping

Abbreviations: $\mathrm{EVA}=$ earliest ventricular activating, $\mathrm{PA}=$ pulmonary artery, $\mathrm{PSC}=$ pulmonary sinus cusp, $\mathrm{PV}=$ pulmonary valve, $\mathrm{PVC}=$ premature ventricular contraction, $\mathrm{PVVJ}=$ pulmonary valve-ventricle junction, RVOT=right ventricular outflow tract, VA=ventricular arrhythmia, VT=ventricular tachycardia, $\mathrm{RF}=$ radiofrequency.

Funding: This work was supported by the Clinical Research Center Project of Department of Science and Technology of Guizhou Province [NO.(2017)5405]; the Guizhou Provincial High-level Innovative Talents Project (GZSYQCC[2015]006); the Guizhou Provincial Science and Technology Foundation (No.[2019]1197); the Guizhou Provincial Science and Technology Social Development Project (No.[2018]2794).

\section{Introduction}

Ventricular arrhythmias (VAs) are one of the most common arrhythmias encountered in clinical practice. VAs with left bundle branch block and inferior axis morphology frequently originate from right ventricular outflow tract (RVOT). ${ }^{1}$ Mapping and ablation of RVOT-type VAs by antegrade manipulating catheter in the RVOT has been well established. ${ }^{2}$ It had the highest acute procedural success (93\%) and long-term success rate $(82 \%)$ comparing to other VA origins. ${ }^{3}$ The general concept was that the VA origins are mainly beneath the pulmonary valve $(\mathrm{PV}) .{ }^{4}$ However, myocardial extensions above the $\mathrm{PV}$ are common. ${ }^{5}$ VAs originating from the pulmonary sinus cusps (PSCs) are receiving increasing attention. ${ }^{6}$ They could be underestimated by the interposed PVs, which would blur electric signals and hinder catheter contact. ${ }^{7}$ A recent study introduced 
a reversed U-curve method which ablated 90\% RVOT-type VAs in the PSCs. ${ }^{8}$ The reversed U-curve method trended a higher immediate and long-term success compared with the antegrade method. ${ }^{8-10} \mathrm{~A}$ proof of VA origin is where successful ablation is performed and where ablation results in a better outcome. The general concept of subvalvular origins in RVOT-type VAs was questioned. The data on directly comparing the ablation effectiveness between the antegrade and reversed U-curve method were limited.

We hypothesized that many origins of idiopathic RVOT-type VAs are above the PV. The ablation effectiveness by reversed U curve and antegrade method are different according to the origins. In this prospective single-center open-label randomized controlled trial, we aimed to investigate the distribution of earliest ventricular activation (EVA) sites in patients with idiopathic RVOT-type VAs and compare the initial ablation success rate of the reversed U-curve method and the antegrade method.

\section{Method}

\section{Study population}

Symptomatic patients with monomorphic RVOT-type VAs and refractory to at least one antiarrhythmic agent were eligible. Patients were excluded if they had structural heart disease or successful ablation outside RVOT. Written informed consents were provided before the procedures. The study was approved by the ethics committee of Guizhou Provincial People's Hospital.

\section{Study design}

The present study is a prospective single-center open-label randomized controlled trial. The study design was illustrated in Figure 1. After activation mapping of RVOT, PSCs and pulmonary artery (PA), the patients were randomly assigned to initial ablation (IA) with the supravalvular strategy using the reversed U-curve method and the subvalvular strategy using the antegrade method. Randomization was performed by tossing a coin. Ablation attempts were performed with uniformed power of $30 \mathrm{w}$, duration of $10 \mathrm{~s}$, and irrigation rate of $17 \mathrm{ml} / \mathrm{min}$. Effective ablation was defined as suppression of VA by the ablation attempts. Isoproterenol was administrated to increase the frequency of premature ventricular contraction if necessary. Once effective ablation was identified, ablation was continued for $60 \mathrm{~s}^{\sim} 90 \mathrm{~s}$. Each ablation application, including ablation attempts, was counted. Successful ablation was defined as non-inducibility of the clinical VAs with isoproterenol elicitation for $30 \mathrm{~min}$. IA success was defined as the successful ablation within the first three ablation applications by the assigned strategy. IA failure included the following situations. Firstly, successful ablation was not achieved with more than three ablation attempts by the assigned strategy. Secondly, the IA targets and EVA sites were contralateral to the PVs, and the distance from EVA site to PVVJ was more than 10mm. Thirdly, the operator had the discretion to declare IA failure after at least one ablation application for the patients whose targets and EVA sites were contralateral to the PVs. Patients with IA failure were managed with the other ablation strategy.

\section{Study procedure}

Antiarrhythmic medications were stopped for at least five half-lives before the procedure. Isoproterenol $1^{\sim} 5$ $\mu \mathrm{g} / \mathrm{min}$ was administered intravenously to provoke the clinical VAs if necessary. An $8 \mathrm{~F}$ saline irrigating catheter (Thermocool, Biosense Webster, Diamond Bar, CA, USA) was advanced to the RVOT region under fluoroscopy through the femoral vein. A long sheath (SL1, Swartz or Preface) was used to facilitate catheter stability. For mapping of the PSCs, the reversed U-curve of the catheter was created in the bifurcation of PA, then pulled back into the PSCs, and confirmed by angiography by injecting contrast through the irrigating tube of the catheter (Figure 2). Pulmonary valve-ventricle junction (PVVJ) was determined by the resistance while retracting the catheter. Before ablation, the angiography was performed again to confirm the catheter's location.

Activation mapping was completed by a three-dimensional mapping system (CARTO 3, Biosense Webster, Diamond Bar, CA, USA). Bipolar and unipolar electrograms were filtered at 30 to $500 \mathrm{~Hz}$ and 0.05 to 400 Hz. Local activating time (LAT) was determined by the correlation of earliest rapid downstroke of unipolar signal with the first sharp peak of the bipolar electrogram. The earliest ventricular activation (EVA) site had 
the longest time interval from LAT to ECG reference. The distance from EVA site to PVVJ was measured on CARTO3. IA targets were selected from the EVA sites with the steepest Q waves in the unipolar and bipolar electrogram. ${ }^{11}$ If the assigned strategy could not engage the target, the less earlier and closest sites on the PVVJ were alternatives.

\section{Study outcome}

The primary outcome was IA success and ablation applications. Secondary outcomes included immediate success, periprocedural complications, and VA recurrence. Clinical PVC frequency more than $10 \%$ of baseline or any episode of VT documented by 24-hours Holter was termed VA recurrence. ${ }^{12}$ An independent events committee judged all adverse events and their relation to ablation.

\section{Follow-up}

All patients were followed up in the cardiology clinic at 1,3,6, and 12 months after the procedure. 24-hour Holter monitoring was scheduled on each follow-up visit.

\section{Statistical analysis}

The sample size was estimated by the time to VA elimination since ablation and ablation applications. ${ }^{7,10,13}$ The average IA successful rate (?) was assumed $85 \%$ and the difference (?) was assumed $20 \%$. For a power of $90 \%\left(\mathrm{Z}_{?}=1.28\right)$ at a two-sided alpha level of $0.05\left(\mathrm{Z}_{?}=1.96\right), 134$ patients were needed. Assuming a dropout rate of $20 \%, 160$ patients were planned to be enrolled. Due to the significant subgroup effect, after reviewing the data, the ethics committee and the leadership discontinued the trial in July 2020.

The primary and secondary outcome was analyzed using the intention-to-treat (ITT) principle. The logistic regression model using frontward selection (Wald) was applied to test the multiplicative interaction between the EVA sites and strategies. Their addictive interaction of absolute excess risk due to interaction (AERI) was calculated. ${ }^{14}$ Continuous variables were expressed as mean \pm standard deviation or median (interquartile range). Categorical variables were expressed as number (frequency \%). For comparison of patient characteristics, differences of continuous variables were tested using Student's t-test if normally distributed; otherwise, Mann-Whitney U test was used. Categorical variables were compared using Fisher exact test. The VA recurrence was compared based on a time-to-first-event analysis using the log-rank test. A two-tailed P value of less than 0.05 was considered statistically significant. For multiple comparisons of 6 contrasts among subgroups based on the EVA sites and IA strategies, a two-tailed P value of less than 0.0083 was considered statistically significant using Bonferroni correction. All statistical analysis was performed using SPSS 26.

\section{Results}

\section{Patient Population and Characteristics}

83 patients were eligible between November 2018 and June 2020 (Figure 3). 10 patients withdrew consents, and 12 had successful ablation in the left ventricular outflow tract. 33 patients assigned to supravalvular strategy and 28 assigned to subvalvular strategy were included in the analysis. Baseline characteristics between the two groups were similar (Table 1). 34/61(55.7\%) of the EVA sites were above PV, and 33/34(97.1\%) were within $10 \mathrm{~mm}$ above PVVJ (Figure $4 \mathrm{~A}$ ). The overall EVA-site distribution was not significantly different $(\mathrm{P}=0.447)$.

\section{Primary outcomes}

IA success rate was $25 / 33(75.8 \%)$ by supravalvular strategy and $16 / 28(57.1 \%)$ by subvalvular strategy $(\mathrm{P}=0.172)$ (Table 2). Supravalvular strategy trended fewer ablation applications as compared to the subvalvular strategy $[1.0(1.0,3.5)$ vs. $2.0(2.0,4.0), \mathrm{P}=0.060]$ (Table 3$)$. The operators declared IA failure in 2 patients assigned to subvalvular strategy. One had the EVA site $18.6 \mathrm{~mm}$ above PVVJ in the PA without IA application. The other had the EVA site $6.5 \mathrm{~mm}$ above PVVJ with 2 unsuccessful IA applications. The EVA sites with IA success or failure showed an ipsilateral or contralateral propensity to IA strategy (Figure $4 \mathrm{~A} \& \mathrm{~B})$. 


\section{Secondary outcomes}

The 20 patients with IA failure all had successful ablation by the other strategy. 1 patient assigned to supravalvular strategy suffered from tamponade after the procedure, but recovered by urgent drainage without blood transfusion. No complications occurred in the other patients. Follow-up continued for a median of 12(10, 12) months until February 2021. In total, 43/61(70.5\%) patients reached the 12 months of followup (Figure 5). 1 patient from supravalvular strategy and 3 from subvalvular strategy had VA recurrence $(\mathrm{P}=0.243)$. The patient from supravalvular strategy had a second procedure after 1 month. Activation mapping revealed the same EVA site in LC, and ablation using the reversed U-curve eliminated PVC within seconds. The patient was then followed for 9 months. No VA recurrence was noted.

\section{Subgroup analysis}

The treatment effect was distinct between the patients with supravalvular and subvalvular EVA sites. For multiple-comparison, either strategy was superior in IA success rate to treat its ipsilateral EVA sites as compared with contralateral ones $(\mathrm{P}<0.0083)$ (Table 2$)$. In the logistic regression model, patient sex, age, presence of VT, PVC frequency, sharp potential reversal at target, EVA site (supravalvular or subvalvular), initial ablation strategy, RVOT diameter, and PA diameter were included to adjust clinical confounders (Table 2). The EVA site was the only baseline characteristic with a remarkable multiplicative treatment interaction $\left(\mathrm{P}_{\text {interaction }}<0.001\right)$. The AERI was calculated as $95.0 \%+85.7 \%-46.7 \%-28.6 \%=105.2 \%$, suggesting a large magnitude of super-addictive interaction between the EVA site and its ipsilateral strategy. In patients with supravalvular EVA sites, supravalvular strategy was associated with a lower risk of IA failure (HR 0.021, 95\% CI 0.002-0.214; $\mathrm{P}=0.001)$. Whereas in patients with subvalvular EVA sites, the supravalvular strategy was associated with a higher risk of IA failure (HR 7.000, 95\% CI 1.098-44.607; P=0.039). The supravalvular strategy had fewer ablation applications in treating supravalvular EVA sites than subvalvular EVA sites, as well as the subvalvular strategy $(\mathrm{P}<0.0083)$ (Table 3$)$.

\section{Analysis of the patients with non-ipsilateral matching}

27 patients with contralateral IA underwent further analysis (Figure 6A). Ablation attempts were effective in 19/27 (70.4\%) patients (Figure 6B). But IA success rate was only 10/27 (37.0\%). By measuring the distances from EVA sites to PVVJ, $5.7 \mathrm{~mm}$ was the maximum for contralateral IA to be effective, indicating the largest heating area of ablation attempts. Using the distance of $5.7 \mathrm{~mm}$, excluding 1 patient with PA origin, effective ablation was estimated $54 / 60(90.0 \%)$ by the supravalvular strategy and $52 / 60(86.7 \%)$ by the subvalvular strategy in the total patients. (Figure 6C).

\section{Discussion}

\section{Major findings}

We reported the first randomized controlled trial, which had investigated the EVA site distribution and compared the ablation effectiveness between the supravalvular strategy and the subvalvular strategy in patients with idiopathic monomorphic RVOT-type VAs. Half of the EVA sites were above the PVs, and most were within $10 \mathrm{~mm}$ above PVVJ. The two strategies did not differ in the IA success rates, ablation applications, complications, and long-term VA recurrence. However, the EVA site was an essential characteristic to determine the ablation effectiveness of the two strategies. Ipsilateral IA remarkably increased the success rate and reduced ablation applications.

\section{Comparison of the strategies}

By determining the PVVJ, we found that half of the RVOT-type VAs were of supravalvular origin. The result is supported by previous studies using intracardiac echocardiography (ICE) and PA angiography. ${ }^{7,8}$ Therefore, it is essential to remind the importance of PVs in catheter ablation of RVOT-type VAs. They protect supravalvular origins when using the antegrade method and prevent engaging subvalvular origins when using the reversed U-curve method. Although the trial did not reach the enrollment goal and the ITT analysis showed no difference, the subgroup analysis had revealed the significant and qualitative interaction 
between the EVA sites and IA strategies. The subgroup effect cannot be explained by chance because of its substantial magnitude, statistical significance, consistency through subgroups, and electrophysiologic rationale. ${ }^{15}$ The result identified the EVA site as a critical baseline characteristic for selecting optimal IA strategy to achieve higher ablation effectiveness. The findings decreased the value of comparing the overall IA success rates between the strategies irrespective of the EVA sites. Since the contralateral IA was no longer in patients' interests and a larger sample size would not add value to the ITT analysis, the ethnic committee and the leadership discontinued the study.

\section{Clinical implication}

One critical electrophysiologic principle is that ablation is more likely to succeed at the target closer to the origin. The high IA success rate by ipsilateral IA indicated the close relationship of the EVA sites and origins. Currently, no ECG characteristics could distinguish RVOT-type VAs with supravalvular origins from those with subvalvular origins. ${ }^{9,10,16}$ The widely accepted stepwise strategy is that after one method fails, then consider the other. However, considering the high proportion of supravalvular origins in RVOT-type VAs, activation mapping by single method is not sufficient to reveal the actual EVA sites. One common cause of ablation failure is that the target is not close enough to the origin. A subset of VAs originating from the PSC junction was reported refractory to the reversed U-curve method. ${ }^{17}$ When using the antegrade method, the earliest activation time beneath PV was $13.2 \pm 4.2 \mathrm{~ms}$ earlier, indicating subvalvular origins. For this reason, the antegrade method had better performance. We defined the VA suppression by contralateral ablation as collateral damage. More ablation applications, prolonged ablation, and higher power were usually needed to achieve success. ${ }^{7}$ The collateral damage pattern of ablation may impair the outcomes and increase the risk of complication. Our findings suggested complete activation mapping for locating VA origins, then selecting the optimal method to ipsilateral ablation for higher effectiveness.

\section{Anatomical considerations}

The effective ablation rate was estimated at $90 \%$ by either strategy in the total patients, indicating $10 \%$ of the patients with the EVA sites over $5.7 \mathrm{~mm}$ to PVVJ required a specific strategy. For the patient with the EVA site $8.5 \mathrm{~mm}$ beneath the PVVJ, the subvalvular strategy was preferred (Supplementary Figure 1). For the patient with the EVA site $5.9 \mathrm{~mm}$ above PVVJ, the supravalvular strategy was preferred (Supplementary Figure 2). For the patient with EVA site in PA, either strategy worked (Supplementary Figure 3). A few patients still had IA failure by ipsilateral IA because catheter stability is also a prerequisite for successful ablation. When the VA origins were close to the PVVJ, the two strategies may have equal ablation effectiveness. The strategy with better catheter stability may be preferred.

\section{Discordances}

The overall IA success rate was lower than previous studies. ${ }^{8,10}$ Firstly, the $30 \mathrm{w}$ and 10 s ablation attempts had avoided collateral damage to VA origins from ablation at a distal target. The setting resulted in a low IA success rate in patients with contralateral ablation. Secondly, the proportion of patients with supravalvular EVA site was fewer than Zhang's cohort [(20/33(60.6\%) vs. $72 / 81(88.9 \%)] .{ }^{8}$ Lastly, since the antegrade method is inferior in catheter stability to the reversed U-curve method, the subvalvular strategy was more susceptible to IA failure as more ablation applications were consumed. ${ }^{9}$

\section{Limitations}

Our study had the following limitations. Firstly, the sample size was small. Secondly, the coin-tossing randomization did not prespecify the EVA site distribution for subgroup analysis. Thirdly, the benefits of fewer ablation applications, such as myocardial damage, total ablation time, procedural time, and X-ray exposure, were not evaluated. Fourthly, the aortic sinus of Valsalva and coronary venous system was not routinely mapped. Fifthly, the echocardiography was reassessed in 11/61(18\%) patients after the procedure. No PV dysfunction was noted. Lastly, ICE and high-density mapping were not used.

\section{Conclusions}


In the prospective single-center open-level randomized trial, half of the origins in idiopathic RVOT-type VAs were above the PV, and most were close to the PVVJ. The supravalvular and subvalvular strategies were not different in IA success rate, ablation application, complication, and VA recurrence. However, they complement locating the EVA sites and facilitate the ipsilateral ablation, which produces a significantly higher success rate.

\section{References}

1. Marcus GM: Evaluation and Management of Premature Ventricular Complexes. Circulation 2020; 141:1404-1418.

2. Kamakura Shiro, Shimizu Wataru, Matsuo Kiyotaka, et al.: Localization of Optimal Ablation Site of Idiopathic Ventricular Tachycardia from Right and Left Ventricular Outflow Tract by Body Surface ECG. Circulation 1998; 98:1525-1533.

3. Latchamsetty R, Yokokawa M, Morady F, et al.: Multicenter Outcomes for Catheter Ablation of Idiopathic Premature Ventricular Complexes. JACC Clin Electrophysiol 2015; 1:116-123.

4. Hutchinson MD, Garcia FC: An organized approach to the localization, mapping, and ablation of outflow tract ventricular arrhythmias. J Cardiovasc Electrophysiol 2013; 24:1189-1197.

5. Gami AS, Noheria A, Lachman N, et al.: Anatomical correlates relevant to ablation above the semilunar valves for the cardiac electrophysiologist: a study of 603 hearts. J Interv Card Electrophysiol 2011; 30:5-15.

6. Liao Z, Zhan X, Wu S, et al.: Idiopathic Ventricular Arrhythmias Originating From the Pulmonary Sinus Cusp: Prevalence, Electrocardiographic/Electrophysiological Characteristics, and Catheter Ablation. J Am Coll Cardiol 2015; 66:2633-2644.

7. Liu CF, Cheung JW, Thomas G, Ip JE, Markowitz SM, Lerman BB: Ubiquitous myocardial extensions into the pulmonary artery demonstrated by integrated intracardiac echocardiography and electroanatomic mapping: changing the paradigm of idiopathic right ventricular outflow tract arrhythmias. Circ Arrhythm Electrophysiol 2014; 7:691-700.

8. Zhang J, Tang C, Zhang Y, Su X: Pulmonary sinus cusp mapping and ablation: A new concept and approach for idiopathic right ventricular outflow tract arrhythmias. Heart Rhythm 2018; 15:38-45.

9. Dong X, Tang M, Sun Q, Zhang S: Usefulness of Reversed U-Curve Technique to EnhanceMapping and Ablation Efficiency in the Treatment ofPulmonary Sinus Cusp-Derived Ventricular Arrhythmias. Am J Cardiol 2018; 122:814-820.

10. Liang Z, Ren X, Zhang T, Han Z, Dong J, Wang Y: Mapping and ablation of RVOT-type arrhythmias: comparison between the conventional and reversed U curve methods. J Interv Card Electrophysiol 2018; $52: 19-30$.

11. Sorgente A, Epicoco G, Ali H, et al.: Negative concordance pattern in bipolar and unipolar recordings: An additional mapping criterion to localize the site of origin of focal ventricular arrhythmias. Heart Rhythm $2016 ; 13: 519-526$.

12. Casella M, Gasperetti A, Gianni C, et al.: Ablation Index as a predictor of long-term efficacy in premature ventricular complex ablation: A regional target value analysis. Heart Rhythm 2019; 16:888-895.

13. Niu G, Feng T, Jiang C, et al.: Predictive value of unipolar and bipolar electrograms in idiopathic outflow tract ventricular arrhythmia mapping and ablation. J Cardiovasc Electrophysiol 2018; 29:900-907.

14. Brankovic M, Kardys I, Steyerberg EW, et al.: Understanding of interaction (subgroup) analysis in clinical trials. European Journal of Clinical Investigation 2019; 49:e13145.

15. Sun X, Ioannidis JPA, Agoritsas T, Alba AC, Guyatt G: How to use a subgroup analysis: users' guide to the medical literature. JAMA 2014; 311:405-411. 
16. Li J, Zheng C, Liu Z-R, et al.: Radiofrequency catheter ablation of ventricular arrhythmias arising from the region above pulmonary valve. BMC Cardiovasc Disord 2019; 19:233.

17. Dong X, Sun Q, Tang M, Zhang S: Catheter ablation of ventricular arrhythmias originating from the junction of the pulmonary sinus cusp via a nonreversed U curve approach. Heart Rhythm 2019; 16:1513-1520.

\section{Figure legends}

\section{Table 1. Patient characteristics at baseline.}

\section{Hosted file}

image1.emf available at https://authorea.com/users/414094/articles/522220-investigatingorigins-of-ventricular-arrhythmia-arising-from-right-ventricular-outflow-tract-andcomparing-initial-ablation-strategies

Table 2. IA success rate.

\section{Hosted file}

image2.emf available at https://authorea.com/users/414094/articles/522220-investigatingorigins-of-ventricular-arrhythmia-arising-from-right-ventricular-outflow-tract-andcomparing-initial-ablation-strategies

Table 3. Ablation applications.

\section{Hosted file}

image3.emf available at https://authorea.com/users/414094/articles/522220-investigatingorigins-of-ventricular-arrhythmia-arising-from-right-ventricular-outflow-tract-andcomparing-initial-ablation-strategies

Figure 1. Study design.

Abbreviations: PVVJ=pulmonary valve-ventricle junction, RVOT=right ventricular outflow tract, $\mathrm{VA}=$ ventricular arrhythmia.

\section{Hosted file}

image4.emf available at https://authorea.com/users/414094/articles/522220-investigatingorigins-of-ventricular-arrhythmia-arising-from-right-ventricular-outflow-tract-andcomparing-initial-ablation-strategies

Figure 2. Illustration of the anatomy and the ablation strategies. (A) The PVVJ was where the PVs adhere to the ventricle wall. The myocardial extensions were the ventricular musculatures that extended above the PVVJ. The yellow star indicated the EVA site. (B) Targeting the EVA site with the antegrade method. (C) The reversed U-curve was created in the bifurcation of PA by curving, advancing, and torquing the ablation catheter. Then the reversed U-curve was loosed to contact the PA wall and retracted into PSC to engage the EVA site.

Abbreviations: $\mathrm{EVA}=$ earliest ventricular activating, $\mathrm{PA}=$ pulmonary artery, $\mathrm{PSC}=$ pulmonary sinus cusp, $\mathrm{PV}=$ pulmonary valve, $\mathrm{PVVJ}=$ pulmonary valve-ventricle junction, $\mathrm{RVOT}=$ right ventricular outflow tract, $\mathrm{VA}=$ ventricular arrhythmia.

\section{Hosted file}

image5.emf available at https://authorea.com/users/414094/articles/522220-investigatingorigins-of-ventricular-arrhythmia-arising-from-right-ventricular-outflow-tract-andcomparing-initial-ablation-strategies

Figure 3. Patient flow and randomization. 
Abbreviations: $\mathrm{EVA}=$ earliest ventricular activating, $\mathrm{PV}=$ pulmonary valve, $\mathrm{PVC}=$ premature ventricular contraction, $\mathrm{RVOT}=$ right ventricular outflow tract, $\mathrm{VA}=$ ventricular arrhythmia.

\section{Hosted file}

image6.emf available at https://authorea.com/users/414094/articles/522220-investigatingorigins-of-ventricular-arrhythmia-arising-from-right-ventricular-outflow-tract-andcomparing-initial-ablation-strategies

Figure 4. Distances from the EVA sites to the PVVJ. The RVOT was arbitrarily divided by STJ, PVVJ, and longitudinal extensions of PSC commissures. The dotted line denoted PVVJ. The markers that coincided with the PVVJ located at the base of PSCs. Y-axis represented the distance from each EVA site to PVVJ. (A) Circles denoted the EVA sites in total patients. (B) The triangles denoted the EVA sites in the patients who had IA success. (C) The crosses denoted the EVA sites in the patients who had IA failure. The black arrow marked the EVA site in PA.

Abbreviations: $\mathrm{AC}=$ anterior cusp, $\mathrm{EVA}=$ earliest ventricular activating, $\mathrm{LC}=$ left cusp, $\mathrm{PA}=$ pulmonary artery, $\mathrm{PSC}=$ pulmonary sinus cusp, $\mathrm{PVVJ}=$ pulmonary valve-ventricle junction, $\mathrm{RC}=$ right cusp, $\mathrm{RV}=$ right ventricle, $\mathrm{RVOT}=$ right ventricular outflow tract, $\mathrm{STJ}=$ sinotubular junction.

\section{Hosted file}

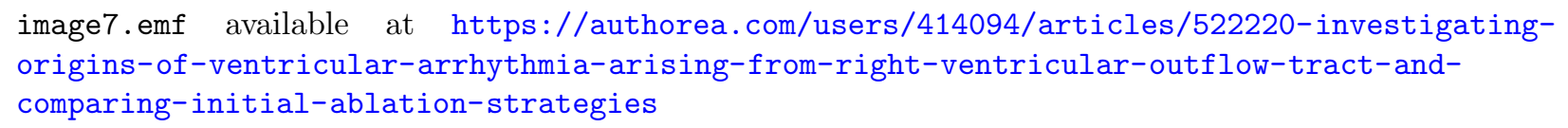

Figure 5. Kaplan-Meier estimates of the accumulative VA recurrence of 12 months. Patient number (frequency) was shown at the bottom. P value was shown on the graph.

Abbreviations: IA=initial ablation, $\mathrm{VA}=$ ventricular arrhythmia.

\section{Hosted file}

image8.emf available at https://authorea.com/users/414094/articles/522220-investigatingorigins-of-ventricular-arrhythmia-arising-from-right-ventricular-outflow-tract-andcomparing-initial-ablation-strategies

Figure 6. Distribution of the EVA sites in patients who had non-ipsilateral-matching IA. (A) The circles denoted the EVA sites with IA success and failure. (B) The circles denoted the EVA sites with ineffective and effective ablation during IA. The black arrow marked the farthest EVA site to PVVJ (-5.7mm) with effective ablation. (C) The circles denoted the EVA sites in total patients. The EVA site (black arrow) in PA was excluded. The dashed box denoted the EVA sites located from $-5.7 \mathrm{~mm}$ to $10.0 \mathrm{~mm}$, estimating that 54/60(90.0\%) patients could have effective ablation by supravalvular strategy. The dotted box denoted the EVA sites from RVOT to $+5.7 \mathrm{~mm}$ above PVVJ, estimating that the subvalvular strategy could have effective ablation in 52/60(86.7\%) patients.

Abbreviations: $\mathrm{EVA}=$ earliest ventricular activating, $\mathrm{IA}=$ initial ablation, $\mathrm{PA}=$ pulmonary artery, PV$\mathrm{VJ}=$ pulmonary valve-ventricle junction.

\section{Hosted file}

image9.emf available at https://authorea.com/users/414094/articles/522220-investigatingorigins-of-ventricular-arrhythmia-arising-from-right-ventricular-outflow-tract-and-

comparing-initial-ablation-strategies 